\title{
Influência da concentração de benzocaína e do comprimento dos peixes na anestesia e na recuperação de tilápias-do-nilo
}

\author{
Daniel Okamura ${ }^{1}$, Felipe Guedes de Araújo ${ }^{1}$, Priscila Vieira e Rosa ${ }^{1}$, Rilke Tadeu Fonseca de \\ Freitas $^{1}$, Luis David Solis Murgas ${ }^{1}$, Marcos Pinto Cesar ${ }^{1}$ \\ ${ }^{1}$ Universidade Federal de Lavras.
}

RESUMO - Com o objetivo de avaliar a influência da concentração de benzocaína na indução e recuperação da anestesia em tilápias-do-nilo (Oreochromis niloticus) de diferentes comprimentos, desenvolveu-se um experimento em blocos casualizados, em esquema fatorial $4 \times 4$, composto de quatro comprimentos de peixes $(6 ; 10 ; 14$ e 18 cm de comprimento total) e quatro concentrações de benzocaína (60; 120; 180 e 240 mg por litro de água). A indução e a recuperação da anestesia foram divididas em três estágios, de acordo com o comportamento dos peixes sob efeito do anestésico, registrando-se o tempo de permanência em cada estágio. As diferenças de comprimento não influenciaram de forma significativa os tempos de anestesia e recuperação. Regressões lineares com plateau foram geradas para estimar o ponto em que o aumento da dose deixou de ter efeito. Durante a indução à anestesia, o plateau ocorreu na concentração de $132 \mathrm{mg} / \mathrm{L}$ e o tempo estimado para atingir a sedação total (estágio 3) foi de 86 segundos. Para permanência do peixe sob sedação total, é necessária concentração de benzocaína de 190 mg/L e o tempo estimado de permanência neste estágio é de 91,1 segundos. Concentrações superiores a $190 \mathrm{mg}$ de benzocaína por litro de água não produzem efeitos que justifiquem seu emprego.

Palavras-chave: anestésico, aquicultura, comprimento, estágio

\section{Effect of benzocaine concentration and fish size on anesthesia and recovery in Nile tilapia}

\begin{abstract}
To evaluate the influence of benzocaine concentration on the induction and recovery from anesthesia in different sized Nile tilapia (Oreochromis niloticus), it was developed a random block experiment design in $4 \times 4$ factorial consisted of four fish lengths (6;10;14 and $18 \mathrm{~cm}$ total lenght) and four benzocaine concentrations (60; $120 ; 180$ and $240 \mathrm{mg}$ of benzocaine per liter of water). The induction and the recovery from anesthesia were divided in three stages based on the behavior of the fishes under anesthetic effect. The time spent in each stage was recorded. Differences in length did not affect significantly the anesthesia and recovery time. Linear plateu regressions were generated to estimate the point which increases on anesthetic doses does not imply in further effects. During the anesthesia induction, the plateu occurred in concentration time of $132 \mathrm{mg} / \mathrm{L}$, and estimated time of 86 seconds would be necessary to achieve the complete sedation (stage 3). For permanence of the fish under total sedation, it is needed $190 \mathrm{~g} / \mathrm{L}$ benzocaine and estimated time of permanence in this stage is 91.1 seconds. Benzocaine concentrations above $190 \mathrm{mg} / \mathrm{L}$ do not produce any additional effects that justify its use.
\end{abstract}

Key Words: anesthetic, aquaculture, length, stage

\section{Introdução}

As formulações com propriedades sedativas mais utilizadas na aquicultura contêm tricaína, benzocaína, quinaldina, metomidato, 2-phenoxietanol, óleo de cravo ou seu ingrediente ativo, o eugenol (Oliveira et al., 2009; Hoskonen \& Pirhonen, 2004; Ross \& Ross, 2000). A escolha do anestésico é influenciada por fatores como preço, disponibilidade e eficiência da droga (Ross \& Ross, 2000).
Um anestésico eficiente tem como características principais atuação rápida (em torno de 3 minutos); curto tempo de recuperação (por volta de 5 minutos); facilidade de sua utilização, com baixos riscos para os animais e o homem; as dosagens devem estar contidas em uma ampla margem de segurança e serem rapidamente metabolizadas e excretadas, não deixando efeitos persistentes nos peixes quando anestesiados sucessivamente (Keene et al., 1998).

A benzocaína é classificada como anestésico local, embora atue de forma sistêmica em peixes, agindo no sistema 
nervoso central. Age bloqueando os canais de sódio, reduzindo os potenciais de ação dos nervos (Arias, 1999). Os efeitos vão desde a leve sedação até o colapso medular (Holloway et al., 2004). Por sua baixa solubilidade, necessita ser dissolvida em etanol antes de ser adicionada à água (Noga, 1995). Possui também baixo efeito residual no metabolismo dos peixes. Hayton et al. (1996) observaram em bagre-do-canal (Ictalurus punctatus) que 1\% das doses inicias de benzocaína permaneceram nos tecidos dos peixes. Segundo Burka et al. (1997), não há relatos de toxicidade em mamíferos, com as dosagens de benzocaína utilizadas em peixes, portanto, é uma droga segura.

A capacidade do anestésico em atenuar o estresse em procedimentos de manejo, comuns nas pisciculturas intensivas, é um parâmetro importante para a sua escolha. Cho \& Heath (2000) ao discutirem os resultados obtidos com dois anestésicos para Oncorhynchus tshawytscha, sugeriram que o aumento dos níveis de cortisol está relacionado à dose utilizada. Barton \& Iwama (2000) afirmaram que as altas dosagens, capazes de provocar rápida imobilização, não elevariam os níveis plasmáticos de cortisol.

As tilápias fazem parte do terceiro maior grupo de peixes cultivados (ciclídeos) no mundo (El-Sayed 1999). No Brasil a produção em 2003 alcançou 64.857 toneladas representando 37,8\% dos peixes cultivados (FAO, 2003).

Objetivou-se determinar uma dose ótima de benzocaina para tilápias-do-nilo de diferentes comprimentos.

\section{Material e Métodos}

Foram utilizados 384 machos de tilápia-do-nilo (Oreochromis niloticus) sexados, obtidos por reprodução natural na estação de piscicultura da Universidade Federal de Lavras. O processo de sexagem dos peixes foi realizado individualmente em cada animal por avaliação da papila urogenital.

O experimento foi desenvolvido em delineamento de blocos casualizados, utilizando-se um ensaio fatorial para avaliar quatro doses de benzocaína (60; 120; 180 e 240 mg de benzocaína por litro de água) aplicada em peixes de quatro comprimentos: $6 \pm 0,6 ; 10 \pm 0,68 ; 14 \pm 1,6$ e $18 \pm 1,4$ cm de comprimento total (5,15 \pm 0,75; 15,28 \pm 1,13; $81,07 \pm 1,09 ; 172,11 \pm 1,75$ g, respectivamente). O experimento foi composto de 16 tratamentos ( 4 doses $\times 4$ comprimentos) e 4 blocos, totalizando 64 parcelas. Em cada parcela foram observados seis peixes de maneira individual. Adotou-se o modelo estatístico representado por:

$$
\mathrm{Y}_{\mathrm{jik}}=\mu+\mathrm{b}_{\mathrm{j}}+\mathrm{c}_{\mathrm{i}}+\mathrm{d}_{\mathrm{k}}+\mathrm{cd}_{\mathrm{ik}}+\mathrm{e}_{\mathrm{jik}}
$$

em que: $Y_{j i k}=$ valor observado da variável resposta referente à parcela jik; j = 1, 2, 3 e 4 blocos; i = 1, 2, 3 e 4 comprimento total; $\mathrm{k}=1,2,3,4$ dosagens de benzocaína; $\mu$ = média geral estimada; $b_{j}=$ efeito do bloco $\mathrm{j}$; $c_{\mathrm{i}}=$ efeito do comprimento do peixe; $\mathrm{d}_{\mathrm{k}}=$ efeito da dosagem da benzocaína $\mathrm{k} ; \mathrm{cd}_{\mathrm{ik}}=$ efeito da interação entre o comprimento do peixe i e a dosagem $\mathrm{k}$; $\mathrm{e}_{\mathrm{jik}}=$ erro experimental, com média zero e variância constante $\mathrm{s}^{2}$.

Cada repetição ou bloco foi precedido de uma etapa de triagem, na qual os peixes foram separados em quatro grupos de acordo com o comprimento. Ao fim de cada triagem, os peixes foram mantidos por uma semana para aclimatação em tanques de alvenaria antes de serem submetidos ao estudo com o anestésico. Esse intervalo foi determinado no intuito de permitir que os peixes se recuperassem do estresse causado pelo manejo da triagem. Os peixes foram alimentados à vontade com ração comercial (28\% de proteína bruta).

O procedimento de anestesia foi realizado em aquários de vidro com capacidade para 15 litros de água, preenchidos com 10 litros de água. As dosagens de benzocaína foram preparadas em volume constante de etanol (100 mL) e adicionadas em uma proporção de $10 \mathrm{~mL}$ de solução por litro de água. Os tratamentos foram ministrados em aquários, cada um contendo um peixe. Esse processo foi realizado seis vezes, completando os seis peixes de cada parcela.

A anestesia e a recuperação dos peixes foram divididas em três fases que se distinguiram por um conjunto de sinais peculiares a cada uma. O tempo de permanência dos peixes em cada estágio durante a anestesia e a recuperação foram cronometrados. Foram adotadas três das quatro fases propostas por Small (2003), descartando-se a quarta fase, referente ao colapso medular (Tabela 1).

Após a avaliação da anestesia, os peixes foram imediatamente transferidos para outro recipiente contendo 50 litros de água sem a solução de etanol e benzocaína para avaliação da recuperação da anestesia. A água utilizada na anestesia e na recuperação foi bombeada de um tanque de decantação, filtrada em filtro de areia e esterilizada com radiação ultravioleta. A temperatura $\left(25 \pm 1,4^{\circ} \mathrm{C}\right)$ e a oxigenação $(6,5 \pm 0,8 \mathrm{dL} / \mathrm{L})$ foram mantidas por meio de termostato e aeração constante. A mortalidade foi contabilizada durante todo o período experimental, tanto na anestesia quanto na recuperação dos peixes.

Os dados obtidos foram analisados pelo software Sistema para Análise Estatística e Genética (UFV, 1997) utilizando-se o teste Scott-Knott a 5\% de probabilidade para comparação das médias. Foi gerado um modelo 
Tabela 1 - Características comportamentais de tilápias em três estágios de indução e três estágios de recuperação à anestesia

\begin{tabular}{|c|c|c|}
\hline Estágio & Indução & Recuperação \\
\hline 1 & $\begin{array}{l}\text { Movimento natatório reduzido, reação a } \\
\text { estímulos externos e equilíbrio normal }\end{array}$ & $\begin{array}{l}\text { Leve recuperação do movimento } \\
\text { opercular e movimentos natatório }\end{array}$ \\
\hline 2 & $\begin{array}{l}\text { Perda do movimento muscular e do } \\
\text { equilíbrio, redução do movimento } \\
\text { opercular e dos reflexos a estímulos externos }\end{array}$ & $\begin{array}{l}\text { Recuperação do equilíbrio e leve } \\
\text { reação a estímulos externos }\end{array}$ \\
\hline 3 & $\begin{array}{l}\text { Perda total dos reflexos a estímulos } \\
\text { externos e movimento opercular quase ausente }\end{array}$ & $\begin{array}{l}\text { Movimento e equilíbrio } \\
\text { natatório normais }\end{array}$ \\
\hline
\end{tabular}

Adaptado de Small (2003).

descontínuo LRP - Linear Response Plateau com estimativa de uma equação de regressão para descrever o comportamento em cada fase da anestesia e recuperação.

\section{Resultados e Discussão}

Não houve mortalidade em nenhuma das doses de benzocaína. O comprimento dos peixes não influenciou $(\mathrm{P}>0,05)$ o comportamento dos animais durante a anestesia e a recuperação. A interação entre comprimento e dosagem também não foi significativa $(\mathrm{P}>0,05)$. De forma semelhante, Walsh \& Pease (2002) constataram que o comprimento do peixe não influenciou na anestesia com a benzocaína em Anguila reinhardtii. No entanto, Olsen (1995) observou em seu estudo efeito significativo no comprimento dos peixes.

$\mathrm{Na}$ maioria das pesquisas com anestésicos, são utilizados diferentes estágios de indução à anestesia como parâmetro de eficiência. Entretanto, apesar de sua grande importância prática, os estágios de recuperação dificilmente são explorados.

As variações na concentração de benzocaína promoveram alterações $(\mathrm{P}<0,05)$ no comportamento dos animais durante o processo de anestesia e de recuperação. De modo geral, com o aumento da dose, a transição entre os estágios de anestesia foi mais rápida (Tabela 2).

Os peixes anestesiados com $60 \mathrm{mg} / \mathrm{L}$ de benzocaína permaneceram por 56 segundos na fase inicial, até o aparecimento dos primeiros sintomas relacionados a anestesia. As outras três dosagens (120, 180 e 240 mg/L), que não apresentaram diferença significativa $(\mathrm{P}<0,05)$, resultaram nessa fase em tempos menores de permanência (30, 30 e 23 segundos, respectivamente).

No estágio 1, os peixes anestesiados com a dosagem de $60 \mathrm{mg} / \mathrm{L}$ permaneceram por tempo maior (174 segundos) que o observado nas concentrações 120, 180 e 240 mg/L, (47, 31 e 27 segundos, respectivamente) antes de finalmente passarem para o estágio seguinte.
Os animais tratados com 180 e 240 mg/L de benzocaína mantiveram-se no segundo estágio por tempo significativamente menor. Os peixes tratados com 120 mg de benzocaína permaneceram por 70 segundos no estágio 2. Apesar de bem menor que o obtido com $60 \mathrm{mg}$ (220 segundos), esse tempo correspondeu ao dobro do tempo gasto pelos animais anestesiados com as dosagens de 180 e $240 \mathrm{mg}$ (35 e 27 segundos, respectivamente) para atingirem a insensibilização total (estágio 3 da insensibilização).

A liberação dos corticosteroides e catecolaminas, relacionada à ação do anestésico, concentra-se no estágio 2 de anestesia, portanto, quanto mais breve a passagem por esta etapa, menor seria a liberação dessas substâncias pelo organismo (Olsen et al., 1995; Rothwell et al., 2005). Caso o objetivo do emprego do anestésico seja mitigar os efeitos do estresse em determinada situação, a utilização da dose correta torna-se fundamental para que o efeito obtido não seja o oposto do almejado.

No intervalo de tempo entre a exposição à benzocaína e a manifestação dos sinais característicos do estágio 3 de anestesia, aqui designado tempo total, o tempo foi de 394 segundos (6,57 minutos) para a dosagem $60 \mathrm{mg} / \mathrm{L}$. Esse tempo é reduzido, notavelmente se a dose for aumentada

Tabela 2 - Período de permanência dos peixes nos estágios de insensibilização e tempo total gasto para a completa indução à anestesia

\begin{tabular}{ccccc}
\hline $\begin{array}{c}\text { Benzocaína } \\
\text { (mg/L } \\
\text { de água) }\end{array}$ & $\begin{array}{c}\text { Início }^{1} \\
\text { (segundos) }\end{array}$ & \multicolumn{2}{c}{$\begin{array}{c}\text { Estágio de anestesia } \\
\text { (segundos) }\end{array}$} & $\begin{array}{c}\text { Tempo } \\
\text { total } \\
\text { (segundos) }\end{array}$ \\
\cline { 2 - 4 } & & 1 & 2 & \\
\hline $60 \mathrm{mg}$ & $56 \mathrm{a} \pm 35$ & $174 \mathrm{a} \pm 80$ & $220 \mathrm{a} \pm 111$ & $394 \mathrm{a} \pm 109$ \\
$120 \mathrm{mg}$ & $30 \mathrm{~b} \pm 13$ & $47 \mathrm{~b} \pm 20$ & $70 \mathrm{~b} \pm 24$ & $117 \mathrm{~b} \pm 34$ \\
$180 \mathrm{mg}$ & $30 \mathrm{~b} \pm 11$ & $31 \mathrm{~b} \pm 12$ & $35 \mathrm{c} \pm 15$ & $66 \mathrm{c} \pm 21$ \\
$240 \mathrm{mg}$ & $23 \mathrm{~b} \pm 7$ & $27 \mathrm{~b} \pm 8$ & $27 \mathrm{c} \pm 14$ & $54 \mathrm{c} \pm 17$ \\
$\mathrm{CV}(\%)$ & 57 & 60 & 65 & 37 \\
\hline
\end{tabular}

Médias na mesma coluna seguidas de letras diferentes diferem $(\mathrm{P}<0,05)$ entre $\mathrm{s}$ pelo teste Scott-Knott.

${ }^{1} \mathrm{O}$ período denominado início refere-se ao tempo gasto para o aparecimento dos primeiros sintomas relacionados à ação do anestésico.

2 Tempo total = estágio 1 da anestesia + estágio 2 da anestesia 
para 120 e $180 \mathrm{mg} / \mathrm{L}$, porém esta tendência não se manteve quando aumentada a dosagem de 180 para $240 \mathrm{mg} / \mathrm{L}$.

Os tempos observados para a anestesia estão de acordo com os relatos de Dawson \& Gilderhus (1979), citados por Stehly \& Gingerich (1999), que indicam doses de 80 a $200 \mathrm{mg}$ de benzocaína/L como o ideal para se alcançar a anestesia total em um período menor que três minutos. Roubach et al. (2005) anestesiaram tambaquis (Colossoma macropomum) com uma dosagem de 100 mg de benzocaína/L e encontraram resultados semelhantes ao deste estudo com $120 \mathrm{mg}$ de benzocaína, de 2,5 minutos para mínimo de movimento opercular.

Os dados referentes à permanência no estágio 3 de anestesia estão expostos juntamente com os das outras fases da recuperação, pois, logo que apresentaram os sinais característicos desse estágio de anestesia, os animais foram transferidos para os aquários de recuperação para serem avaliados. O tempo contabilizado para esse período referese ao tempo que os peixes levaram até apresentarem os primeiros movimentos operculares e das nadadeiras após serem retirados do banho de anestésico (Tabela 3).

O terceiro estágio da anestesia representa o limite entre a anestesia reversível e o colapso medular que leva o animal a óbito. É também a fase em que os animais permanecem sob o maior efeito da droga, apresentando alto grau de insensibilização.

Os peixes sedados com $60 \mathrm{mg}$ de benzocaína por litro de água apresentaram menor tempo de permanência no terceiro estágio $(\mathrm{P}<0,05)$, permanecendo 30 segundos nesta fase. Os animais anestesiados com 120 mg de benzocaína apresentaram tempo maior em comparação aos tratados com 60 mg e menor em relação aos tratados com 180 e $240 \mathrm{mg} / \mathrm{L}(\mathrm{P}<0,05)$, os quais apresentaram tempos de 93 e 89 segundos, respectivamente.

Durante o primeiro estágio de recuperação, as dosagens de benzocaína estudadas não diferiram significativamente,

Tabela 3 - Tempo de permanência nos estágios de recuperação à anestesia e tempo total gasto para completa recuperação dos peixes

\begin{tabular}{ccccc}
\hline \multirow{2}{*}{$\begin{array}{c}\text { Benzocaína } \\
\text { (mg/L } \\
\text { de água) }\end{array}$} & $\begin{array}{c}\text { Estágio da } \\
\text { anestesia }\end{array}$ & $\begin{array}{c}\text { Estágio de recuperação } \\
\text { (segundos) }\end{array}$ & \multirow{2}{*}{$\begin{array}{c}\text { Tempo } \\
\text { (segundos) }\end{array}$} & $\begin{array}{c}\text { total } \\
\text { (segundos) }\end{array}$ \\
\cline { 3 - 4 } & & 1 & 2 & \\
\hline $60 \mathrm{mg}$ & $30 \mathrm{a} \pm 13$ & $69 \mathrm{a} \pm 25$ & $217 \mathrm{a} \pm 91$ & $286 \mathrm{a} \pm 96$ \\
$120 \mathrm{mg}$ & $58 \mathrm{~b} \pm 19$ & $71 \mathrm{a} \pm 22$ & $209 \mathrm{a} \pm 80$ & $280 \mathrm{a} \pm 87$ \\
$180 \mathrm{mg}$ & $93 \mathrm{c} \pm 53$ & $66 \mathrm{a} \pm 28$ & $171 \mathrm{~b} \pm 82$ & $237 \mathrm{~b} \pm 93$ \\
$240 \mathrm{mg}$ & 89c \pm 45 & $63 \mathrm{a} \pm 24$ & $151 \mathrm{~b} \pm 82$ & $214 \mathrm{~b} \pm 99$ \\
$\mathrm{CV}(\%)$ & 54 & 37 & 44 & 36 \\
\hline
\end{tabular}

Médias na mesma coluna seguidas de diferentes letras diferem $(\mathrm{P}<0,05)$ entre si pelo teste de Scott-Knott.

${ }^{1}$ Tempo total = estágio 1 da recuperação + estágio 2 da recuperação. comprovando que, após a recuperação dos primeiros movimentos, os peixes levaram o mesmo tempo para atingirem o segundo estágio.

No segundo estágio da recuperação, os peixes tratados com as dosagens mais altas (180 e $240 \mathrm{mg} / \mathrm{L}$ ) apresentaram tempo de permanência de 171 e 151 segundos, resultados numericamente inferiores aos obtidos com as doses menores (60 e $120 \mathrm{mg} / \mathrm{L}$ ), de 217 e 209 segundos, respectivamente.

Esse maior tempo de permanência na segunda fase observado para as doses menores contribuiu para que o tempo total da recuperação fosse maior que o observado nas dosagens de 180 e 240 mg de benzocaína, em que os peixes retomaram o equilíbrio e a percepção aos estímulos externos mais rapidamente $(\mathrm{P}<0,05)$, apresentando tempo total de 237 e 214 segundos, respectivamente.

De modo geral, todas as dosagens de benzocaína estudadas proporcionaram tempo de recuperação compatível com o limite ideal para um anestésico sugerido por Keene et al. (1998), de 300 segundos. No entanto, os grupos sedados com 60 e 120 mg de benzocaína foram os que mais se aproximaram desse limite, apresentando $286 \mathrm{e}$ 280 segundos, respectivamente. Esses resultados se assemelham aos de Roubach et al. (2005), que observaram recuperação total em 275 segundos ao utilizarem concentração de 100 mg/L de benzocaína em tambaqui.

A redução do tempo entre as fases da anestesia com o aumento da dose do anestésico e estimativa dos pontos de plateau indicam que é possível determinar o ponto em que essa relação dose/tempo deixa de ser válida, ou seja, quando o aumento da dose deixa de influenciar os tempos de permanência em cada estágio (Figura 1).

No estágio 1 da anestesia, o plateau ocorre na dose de $128 \mathrm{mg} / \mathrm{L}$, para qual se tem um tempo de 26,8 segundos; na segunda etapa da anestesia, a dose que marca o ponto de plateau é $128,6 \mathrm{mg} / \mathrm{L}$ e o tempo correspondente é de 55,9 segundos. No terceiro estágio, o ponto é determinado pela dose $132 \mathrm{mg} / \mathrm{L}$, que teria um tempo estimado em 86 segundos (Figura 1a).

Nos dados referentes à recuperação, o plateau da primeira fase da recuperação ocorre na dose de 190 mg/L, o da segunda ocorre com 166,4 mg/L e os tempos correspondentes são 91,1 e 152,4 segundos, respectivamente. O terceiro estágio parece não depender das doses utilizadas (Figura 1b).

Aplicando as informações obtidas com esse modelo de regressão a uma situação prática, pôde-se estimar, por exemplo, que o tempo máximo de sedação é obtido com 190 mg/L de benzocaína; que o estágio três da indução seja 

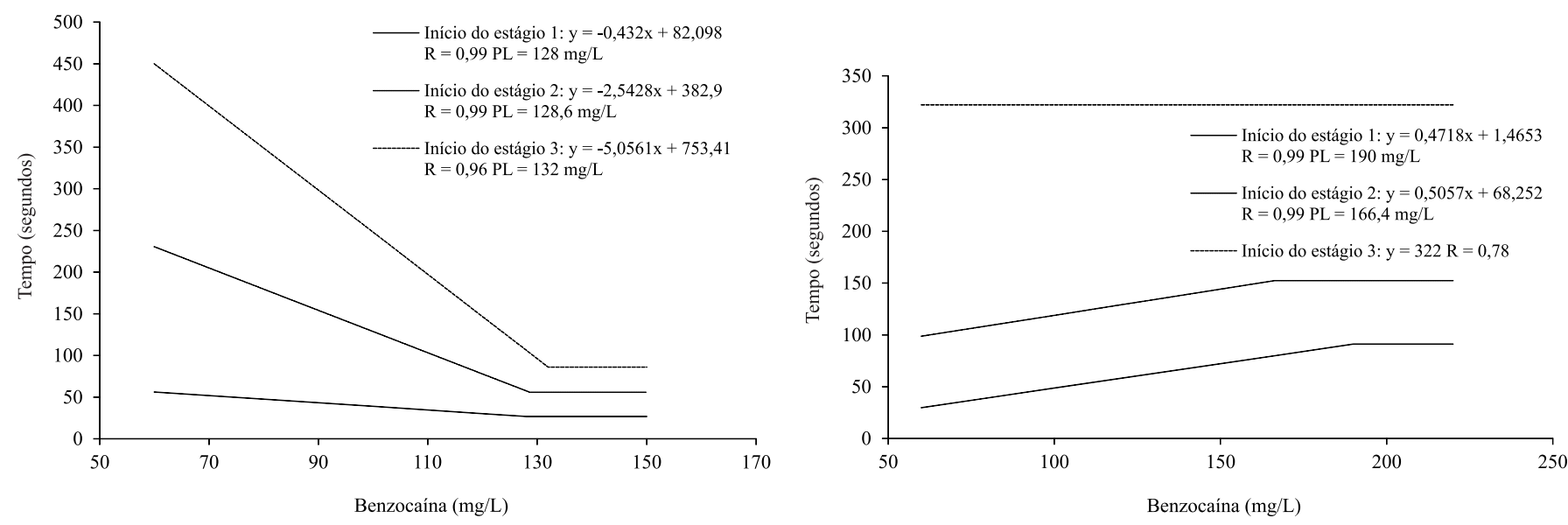

Figura 1 - Tempo de permanência de tilápias-do-nilo em cada estágio da anestesia (a) e em cada estágio da recuperação (b) após imersão em diferentes concentrações de benzocaína.

alcançado após 86 segundos, e que, com esta dosagem, o peixe permanecerá sob a anestesia total (antes de iniciar o primeiro estágio da recuperação) por 91,1 segundos. Essa foi a concentração de benzocaína que proporcionou o maior tempo de permanência dos peixes sob anestesia total e o menor tempo de transição entre os estágios de anestesia e recuperação entre os estágios 1 ao 3.

As regressões encontradas correspondem a resultados obtidos por outros pesquisadores em outras espécies. Antunes et al. (2008) observaram em Cyprinus carpio que a concentração de 125,79 mg de benzocaína por litro de água induz a anestesia total em 114,33 segundos. No entanto, Inoue et al. (2002) buscaram a dose ideal de benzocaína para Brycon cephalus determinaram que concentrações de 40 a $60 \mathrm{mg}$ induzem os peixes ao estágio 3 de anestesia em 396 a 66,0 segundos, respectivamente.

A benzocaína mostrou-se eficaz na anestesia da tilápiado-nilo (Oreochromis niloticus) com 6 a $18 \mathrm{~cm}$ de comprimento. A dose ideal dependerá dos objetivos práticos de sua utilização. Para as situações mais comuns no manejo das pisciculturas, onde geralmente se deseja uma rápida atuação do anestésico evitando o agravamento do estresse e um efeito sedativo mais prolongado, a dosagem de $190 \mathrm{mg} / \mathrm{L}$ pode ser utilizada. Doses maiores não produzem efeitos adicionais que justifiquem a viabilidade de seu emprego.

\section{Conclusões}

A dosagem de 190 mg de benzocaína por litro de água é o suficiente para induzir por 91,1 segundos a anestesia total de tilápias-do-nilo de qualquer dos comprimentos avaliados.

\section{Referências}

ANTUNES, M.I.P.; SPURIO, R.S.; GODOI, D.A. et al. Cloridrato de benzocaína na anestesia de carpa (Cyprinus carpio). Ciências Agrárias, v.29, n.1, p.151-156, 2008.

ARIAS, H.R. Role of local anesthetics on both cholinergic and serotonergic ionotropic. Neuroscience and Biobehavioral Reviews, v.23, p.817-843, 1999.

BARTON, B.A. Salmonid fishes differ in their cortisol and glucose responses to handling and transport stress. North American Journal of Aquaculture, v.62, n.1, p.12-18, 2000.

BURKA, J.F.; HAMMELL, K.L.; HORSBERG, T.E. et al. Drugs in salmonid aquaculture. Journal of Veterinary Pharmacology and Therapeutics, v.20, p.333-349, 1997.

CHO, G.K.; HEATH, D.D. Comparison of tricaíne methanesulphonate (MS222) and clove oil anaesthesia effects on the physiology of juvenile Chinook salmon Oncorhynchus tshawytscha (Walbaum). Aquaculture Research, v.31, p.537-546, 2000.

EL-SAYED, A.-F. M. Alternative dietary protein sources for farmed tilapia, Oreochromis spp. Aquaculture, v.179, p.149-168, 1999.

FAO. The state of world's fhisheries and aquaculture 2002. FAO information division. Rome, 2003. Disponível em: <http// www.fao.org/sof/sofia/index_en.htm>. Acesso em: 12/3/2003.

HAYTON, W.L.; SZOKE, A.; KEMMENOE, B.H. et al. Disposition of benzocaine in channel catfish. Aquatic Toxicology, v.36, p.99-1 13, 1996.

HOLLOWAY, A.C.; KEENE, J.L.; NOAKES, D.G. et al. Effects of clove oil and MS-222 on blood hormone profiles in rainbow trout Oncorhynchus mykiss, Walbaum. Aquaculture Research, v.35, p.1025-1030, 2004.

HOSKONEN, P.; PIRHONEN, J. Temperature effects on anaesthesia with clove oil in six temperate-zone fishes. Journal of Fish Biology, v.64, p.1136-1142, 2004.

INOUE, L.A.K.A.; NETO, C.S.; MORAES, G. Benzocaína como anestésico para juvenis de matrinxã (Brycon cephalus). Boletim Técnico do CEPTA, v.15, p.23-30, 2002.

KEENE, J.I.; NOAKES, D.I.G.; MOCCIA, R.D. et al. The efficacy of clove oil as an anaesthetic for rainbow trout, Oncorhynchus mykiss (Walbaum). Aquaculture Research, v.29, p.89-101, 1998.

NOGA, E.J. Fish disease: diagnosis and treatment. Saint Louis: Mosby-Year Book, 1995. 367p. 
OLIVEIRA, J.R.; CARMO, J.L.; OLIVEIRA, K.K.C. Cloreto de sódio, benzocaína e óleo de cravo-da-índia na água de transporte de tilápia-do-nilo. Revista Brasileira de Zootecnia, v.38, n.7, p.1163-1169, 2009.

OLSEN, Y.A.; EINARSDOTTIR, I.E.; NILSSEN, K.J. Metomidate anaesthesia in Atlantic salmon, Salmo salar, prevents plasma cortisol increase during stress. Aquaculture, v.134, p.155-168, 1995.

ROSS, L.G.; ROSS B. Book Reviews: Anaesthetic and sedative techiniques for aquatic animals. Journal of fish biology, v.56, p.1562-1565, 2000.

ROTHWELL, S.E.; BLACK, S.E.; JERRETT, A.R. et al. Cardiovascular changes and catecholamine release following anaesthesia in Chinook salmon (Oncorhynchus tshawytscha) and snapper (Pagrus auratus). Comparative Biochemistry and Physiology, part A, v.140, p.289-298, 2005.

ROUBACH, R.; GOMES, L.C.; FONSECA, F.A.L. et al. Eugenol as an efficacious anaesthetic for tambaqui, Colossoma macropomum (Cuvier). Aquaculture Research, v.36, p.1056-1061, 2005.

UNIVERSIDADE FEDERAL DE VIÇOSA - UFV. Sistema para análise estatística e genética - SAEG. Manual de uso. Viçosa, MG: Funarbe, 1997. 150p.

SMALL, B.C. Anesthetic efficacy of metomidate and comparison of plasma cortisol responses to tricaine methanesulfonate, quinaldine and clove oil anesthetized channel catfish Ictalurus punctatus. Aquaculture, v.218, p.177-185, 2003.

STEHLY, G.R.; GINGERICH, W.H. Evaluation of AQUI-STM (efficacy and minimum toxic concentration) as a fish anaesthetic/sedative for public aquaculture in the United States. Aquaculture Research, v.30, p.365-372, 1999.

WALSH, C.T.; PEASE, B.C. The use of clove oil as an anaesthetic for the longfinned eel, Anguilla reinhardtii (Steindachner). Aquaculture Research, v.33, p.627-635, 2002. 